\title{
Shared genetic predisposition in rheumatoid arthritis-interstitial lung disease and familial pulmonary fibrosis
}

\author{
Pierre-Antoine Juge 1,2,51, Raphaël Borie 2,3,4,51 , Caroline Kannengiesser $^{2,5,6,51}$, \\ Steven Gazal ${ }^{2,7,8}$, Patrick Revy ${ }^{9,10}$, Lidwine Wemeau-Stervinou ${ }^{11,12}$, \\ Marie-Pierre Debray ${ }^{2,13}$, Sébastien Ottaviani ${ }^{1,2}$, Sylvain Marchand-Adam ${ }^{14,15,16}$, \\ Nadia Nathan ${ }^{17,18,19}$, Gabriel Thabut ${ }^{2,4,20}$, Christophe Richez (10) 21,22,23, \\ Hilario Nunes ${ }^{24,25}$, Isabelle Callebaut ${ }^{19,26}$, Aurélien Justet ${ }^{2,3}$, \\ Nicolas Leulliot ${ }^{10,27}$, Amélie Bonnefond ${ }^{12,28,29}$, David Salgado 30,31 , \\ Pascal Richette $2,32,33$, Jean-Pierre Desvignes ${ }^{30,31}$, Huguette Lioté ${ }^{34}$, \\ Philippe Froguel ${ }^{12,28,35}$, Yannick Allanore ${ }^{10,36,37}$, Olivier Sand ${ }^{12,28,29}$, \\ Claire Dromer ${ }^{23,38}$, René-Marc Flipo ${ }^{12,39}$, Annick Clément ${ }^{17,18,19}$, \\ Christophe Béroud ${ }^{30,31,40}$, Jean Sibilia ${ }^{41,42,43}$, Baptiste Coustet ${ }^{1,2}$, \\ Vincent Cottin ${ }^{44,45}$, Marie-Christophe Boissier ${ }^{25,46,47}$, Benoit Wallaert ${ }^{11,12}$, \\ Thierry Schaeverbeke ${ }^{21,22,23}$, Florence Dastot le Moal ${ }^{17,19,48}$, Aline Frazier ${ }^{2,32}$, \\ Christelle Ménard ${ }^{17,18,19}$, Martin Soubrier ${ }^{49}$, Nathalie Saidenberg ${ }^{25,47}$, \\ Dominique Valeyre ${ }^{24,25}$, Serge Amselem ${ }^{17,18,48}$, the FREX consortium ${ }^{52}$, \\ Catherine Boileau $\mathbb{1}^{2,5,50}$, Bruno Crestani ${ }^{2,3,4}$ and Philippe Dieudé ${ }^{1,2,6}$
}

@ERSpublications

Contribution of TERT, RTEL1, PARN and SFTPC mutations to rheumatoid interstitial lung disease susceptibility http://ow.ly/SXEm30a98Ic

Cite this article as: Juge $\mathrm{P}-\mathrm{A}$, Borie $\mathrm{R}$, Kannengiesser $\mathrm{C}$, et al. Shared genetic predisposition in rheumatoid arthritis-interstitial lung disease and familial pulmonary fibrosis. Eur Respir J 2017; 49: 1602314 [https:// doi.org/10.1183/13993003.02314-2016].

ABSTRACT Despite its high prevalence and mortality, little is known about the pathogenesis of rheumatoid arthritis-associated interstitial lung disease (RA-ILD). Given that familial pulmonary fibrosis (FPF) and RA-ILD frequently share the usual pattern of interstitial pneumonia and common environmental risk factors, we hypothesised that the two diseases might share additional risk factors, including FPF-linked genes. Our aim was to identify coding mutations of FPF-risk genes associated with RA-ILD.

We used whole exome sequencing (WES), followed by restricted analysis of a discrete number of FPFlinked genes and performed a burden test to assess the excess number of mutations in RA-ILD patients compared to controls.

Among the 101 RA-ILD patients included, 12 (11.9\%) had 13 WES-identified heterozygous mutations in the TERT, RTEL1, PARN or SFTPC coding regions. The burden test, based on 81 RA-ILD patients and 1010 controls of European ancestry, revealed an excess of TERT, RTEL1, PARN or SFTPC mutations in RA-ILD patients (OR 3.17, 95\% CI 1.53-6.12; $\mathrm{p}=9.45 \times 10^{-4}$ ). Telomeres were shorter in RA-ILD patients with a TERT, RTEL1 or PARN mutation than in controls $\left(\mathrm{p}=2.87 \times 10^{-2}\right)$.

Our results support the contribution of FPF-linked genes to RA-ILD susceptibility.

This article has supplementary material available from erj.ersjournals.com

Received: Nov 282016 | Accepted after revision: Feb 112017

Copyright @ERS 2017 
Affiliations: ${ }^{1}$ APHP, Hôpital Bichat, Service de Rhumatologie, DHU FIRE, Paris, France. ${ }^{2}$ Université Paris Diderot, Sorbonne Paris Cité, Paris, France. ${ }^{3}$ APHP, Hôpital Bichat, Service de Pneumologie A, DHU FIRE, Paris, France. ${ }^{4}$ INSERM U1152, Paris, France. ${ }^{5}$ APHP, Service de Génétique, Hôpital Bichat, Paris, France. ${ }^{6}$ INSERM, UMR_1149 Centre de Recherches sur l'Inflammation Paris, Paris, France. ${ }^{7}$ INSERM, IAME, UMR 1137, Paris, France. ${ }^{8}$ APHP, Plateforme de génomique constitutionnelle du GHU Nord, Hôpital Bichat, Paris, France. ' INSERM UMR_1163, Laboratory of Genome Dynamics in the Immune System, Institut Imagine, Paris, France. ${ }^{10}$ Université Paris Descartes, Sorbonne Cité, Paris, France. ${ }^{11} \mathrm{CHRU}$ de Lille, Service de Pneumologie et Immuno-Allergologie, Centre de compétence maladies pulmonaires rares, FHU IMMINENT, Lille, France. ${ }^{12}$ Université Lille 2, Lille, France. ${ }^{13} \mathrm{APHP}$, Hôpital Bichat, Service de Radiologie, Paris, France. ${ }^{14} \mathrm{CHRU}$ Tours, Service de Pneumologie, Tours, France. ${ }^{15}$ Université Francois Rabelais, Tours, France. ${ }^{16}$ INSERM, U1100, Tours, France. ${ }^{17}$ APHP, Service de Pneumologie Pédiatrique et Centre de référence des maladies respiratoires rares, Hôpital Trousseau, Paris, France. ${ }^{18}$ INSERM UMR_S933, Paris, France. ${ }^{19}$ Université Pierre et Marie Curie, Sorbonne Paris Cité, Paris, France. ${ }^{20} \mathrm{APHP}$, Hôpital Bichat, Service de Pneumologie B, DHU FIRE, Paris, France. ${ }^{21} \mathrm{CHU}$ de Bordeaux, service de rhumatologie, Bordeaux, France. ${ }^{22}$ Immuno ConcEpT, CNRS UMR_5164, Bordeaux, France. ${ }^{23}$ Université de Bordeaux, Bordeaux, France. ${ }^{24}$ AP-HP, Hôpital Avicenne, Service de Pneumologie, Bobigny, France. ${ }^{25}$ Université Paris 13 , Sorbonne Paris Cité, Paris, France. ${ }^{26}$ CNRS UMR_7590, MNHM-IRDIUC, Paris, France. ${ }^{27}$ Laboratoire de Cristallographie et RMN Biologiques, UMR CNRS 8015 , Faculté de Pharmacie, Paris, France. ${ }^{28} \mathrm{CNRS}$, UMR_8199, Lille, France. ${ }^{29}$ European Genomic Institute for Diabetes, Lille, France. ${ }^{30}$ Aix-Marseille Université, GMGF, Marseille, France. ${ }^{31}$ INSERM, UMR S 910, Marseille, France. ${ }^{32}$ APHP, hôpital Lariboisière, Service de Rhumatologie, Paris, France. ${ }^{33}$ INSERM, UMR_1132, Paris, France. ${ }^{34}$ APHP, Hôpital Tenon, Service de Pneumologie, Paris, France. ${ }^{35}$ Dept of Genomics of Common Diseases, School of Public Health, Imperial College London, Hammersmith Hospital, London, UK. ${ }^{36}$ APHP, Hôpital Cochin, Service de Rhumatologie A, Paris, France. ${ }^{37}$ INSERM, U1016, UMR_8104, Paris, France. ${ }^{38} \mathrm{CHU}$ de Bordeaux, Service d'Imagerie Thoracique et Cardiovasculaire, Pessac, France. ${ }^{39} \mathrm{CHU}$ de Lille, Service de Rhumatologie, Lille, France. ${ }^{40} \mathrm{APHM}$, Hôpital La Timone Enfants, Laboratoire de Génétique Moléculaire, Marseille, France. ${ }^{41} \mathrm{CHRU}$ de Strasbourg, Service de Rhumatologie, Hôpital de Hautepierre, Strasbourg, France. ${ }^{42}$ INSERM UMR_S1109, Laboratoire d'Immuno-Rhumatologie Moléculaire, CRHI, FMTS, Université de Strasbourg, Strasbourg, France. ${ }^{43}$ Fédération Hospitalo-Universitaire OMICARE, Strasbourg, France. ${ }^{44}$ Hospices Civils de Lyon, Hôpital Louis Pradel, Centre national de référence des maladies pulmonaires rares, Lyon, France. ${ }^{45}$ INRA, UMR_754, Université Claude Bernard Lyon 1, Lyon, France. ${ }^{46}$ INSERM U1125, Bobigny, France. ${ }^{47}$ APHP, GH HUPSSD, Service de Rhumatologie, Bobigny, France. ${ }^{48}$ APHP, Département de Génétique, Hôpital Trousseau, Paris, France. ${ }^{49} \mathrm{CHU}$ Clermont-Ferrand, Service de Rhumatologie, INRA, UMR1019, UNH, CRNH Auvergne, Clermont-Ferrand, France. ${ }^{50}$ INSERM, UMR_1148, Maladies structurelles cardiovasculaires, Paris, France. ${ }^{51}$ These authors contributed equally. ${ }^{52} \bar{M}$ embers are listed in the supplementary material.

Correspondence: Philippe Dieudé, Service de Rhumatologie, Hôpital Bichat, 46 rue Henri Huchard, 75018 Paris, France. E-mail: philippe.dieudedaphp.fr

\section{Introduction}

Rheumatoid arthritis (RA) is a destructive, systemic inflammatory and autoimmune disorder that affects up to $1 \%$ of the general adult population worldwide. Extra-articular disease occurs in nearly $50 \%$ of all RA patients, the lung being frequently involved [1]. Indeed, lung disease causes $10 \%-20 \%$ of all deaths in RA patients [2-4]. Specifically, interstitial lung disease (ILD) is the leading cause of mortality, accounting for a mortality rate that is approximately $13 \%$ higher in RA patients as compared to the general population $[2,4,5]$, with a three-fold higher risk of death for those with ILD than those without [5]. In addition, whereas overall RA mortality rates are decreasing, RA-ILD deaths are increasing [6]. Despite its frequency and prognostic impact, RA-ILD has not been given much attention and we are far from understanding its pathogenesis [7].

In comparison to ILD occurring in other connective tissue diseases, patients with RA-ILD frequently present the usual interstitial pneumonia (UIP) pattern, which is characteristic of pulmonary fibrosis [8]. This pattern might explain the poor outcomes of RA-ILD patients, with survival rates similar to those of pulmonary fibrosis patients [9]. Familial pulmonary fibrosis (FPF) that might display histological patterns other than UIP has been linked to mutations in telomere maintenance-associated [10-15] and surfactant protein genes [16-18]. Most importantly, FPF and RA-ILD share common risk factors, such as cigarette smoking and the male sex $[19,20]$.

Given the above-cited similarities of RA-ILD and FPF, we hypothesised that RA-ILD and FPF share genetic risk factors. Therefore, we performed whole exome sequencing (WES) in RA-ILD patients to determine the contribution of mutations in genes previously linked to FPF.

Support statement: This work was supported by research grants from the Société Française de Rhumatologie, Club Rhumatismes Inflammation, la Chancellerie des Universités de Paris (legs Poix), Sorbonne Paris Cité (FPI-SPC Program), Agence Nationale de la Recherche (grants ANR-10-LABX-46, ANR-10-EQPX-07-01, ANR-14-CE10-0006 and ANR-10-INBS-09), France Génomique National Infrastructure, unrestricted grants from Pfizer, Roche and Chugaï, and the Centre de Resources Biologiques Hôpital Bichat, Paris, France. Additional acknowledgements can be found in the supplementary material. Funding information for this article has been deposited with the Crossref Funder Registry.

Conflict of interest: Disclosures can be found alongside this article at erj.ersjournals.com 


\section{Methods}

Study participants

Consecutive RA patients with high-resolution computed tomography (HRCT) chest scans showing ILD were recruited by a French network of ILD-expert pulmonologists and RA-expert rheumatologists from 10 university hospitals during the period 2013-2015. All medical records were centrally reviewed by multidisciplinary discussion that included a pulmonologist (B. Crestani), rheumatologist (P. Dieudé) and radiologist. Medical records were independently reviewed to confirm whether subjects met the American College of Rheumatology criteria for RA [21]. The HRCT chest scans of the subjects were analysed by an experienced reader, blinded to clinical, biologic and genetic data, who scored the scans to ensure that the criteria for ILD were met [9]. In-house subjects $(n=1010)$ without known autoimmune/inflammatory and/ or pulmonary diseases served as healthy controls (supplementary material). The relevant ethics committees approved all procedures, and written informed consent was obtained from all participants in agreement with French bioethics laws.

\section{WES followed by restricted analysis of FPF-linked genes in RA-ILD patients}

FPF-risk genes implicated in telomere maintenance include telomerase reverse transcriptase (TERT) [10], telomerase RNA component (TERC) [10,11], dyskerin (DKC1) [12], telomere-interacting factor-2 (TINF2) [13], regulator of telomere-elongation helicase-1 (RTEL1) $[14,15]$ and polyadenylation-specific ribonuclease deadenylation nuclease $(P A R N)$ [15]. FPF mutations are also found in genes that encode the following surfactant proteins: surfactant protein C (SFTPC) [16], ATP-binding cassette, subfamily A, member 3 (ABCA3) [17] and surfactant protein A2 (SFTPA2) [18]. We used WES, followed by an analysis restricted to these nine FPF-linked genes to assess excess mutations in RA-ILD patients. Sanger sequencing independently confirmed the WES-identified candidate disease-associated mutations.

\section{TERT and RTEL1 molecular modelling and three-dimensional structure visualisation}

Models of the three-dimensional structure of TERT and RTEL1 were built and analysed to assess the mutation effects.

\section{Genotype-phenotype association analyses}

Clinical, demographic, biological, HRCT chest scan and pulmonary function test results were assessed at RA-ILD patient inclusion. All HRCT scans were centrally reviewed and scored by a senior radiologist (M-P. Debray) (supplementary material). A telomeric restriction fragment length (TRFL) assay was used to measure telomere length in RA-ILD patients with mutations in telomere-maintenance candidate genes.

\section{Statistical analyses}

Power calculation

The 101 cases and 1010 controls provided a power higher than $70 \%$ to detect an overall association with an odds ratio of 3.0 (supplementary material).

Ancestry-inference analysis

Ancestry of all RA-ILD patients and controls was verified by principal component analysis, based on the individuals of the 1000 Genomes Project. To avoid population stratification bias, all outlier patient (i.e. those not of European ancestry according to the 1000 Genomes Project) data were excluded from the association analyses (burden test).

\section{Burden test}

A classical burden test was used to assess excess-risk mutations in RA-ILD. Significance was assessed using a one-sided Wald test.

\section{Genotype-phenotype association analysis}

Continuous variables, expressed as median (range), were compared using the t-test; categorical variables, expressed as $\mathrm{n}(\%)$, were compared by the Fisher's exact test. Comparisons of RA-ILD patients with mutations were drawn using non-parametric tests because of the small sample size. Generalised additive models were used to evaluate the linearity of the relationship between continuous variables and mutation probability. Telomere lengths for TERT/RTEL1/PARN mutation carriers $(\mathrm{n}=11)$ and 15 healthy age-matched controls (TRFL being previously assessed for 13 of them [22]) were compared by logistic regression adjusted for age, using the R 3.1.2 glm function and corresponding figures were created with Graphpad Prism 6.0b. All statistical analyses were performed using the R 3.1.2 software. Levels of significance were defined at $\mathrm{p}<0.05$.

Methods and corresponding analyses are detailed in the supplementary material. 


\section{Results}

\section{Phenotype of RA-ILD patients}

We included 101 consecutive independent RA-ILD patients. Mean \pm SD age at RA onset was 53.54 \pm 15.40 years; $82.1 \%$ were anti-citrullinated peptide antibody-positive, 84 . $8 \%$ were rheumatoid factor-positive and $71.1 \%$ had erosive disease. Mean age at ILD onset was $61.42 \pm 11.81$ years and mean RA duration before ILD detection was $7.93 \pm 10.83$ years. Overall, $54.5 \%$ of all patients were ever-smokers and $65.4 \%$ showed the UIP pattern on HRCT. The demographic information and clinical characteristics are summarised in table 1.

\section{Exome sequencing of FPF-linked genes in RA-ILD patients}

WES combined with restricted analysis of the nine FPF-linked genes, followed by Sanger sequencing confirmation revealed that $12 / 101$ RA-ILD patients (11.9\%) carried 13 heterozygous mutations in the TERT, RTEL1, PARN or SFTPC coding regions (table 2, figure S2).

For telomere-maintenance genes, six RA-ILD patients carried six heterozygous TERT mutations: c.2383-2A $>\mathrm{G}$, affecting intron splicing, not reported in the Exome Aggregation Consortium (ExAC) database, and c.3323C $>$ T, p.Pro1108Leu, with ExAC minor allele frequency (MAF) of $5.55 \times 10^{-5}$. In addition, four RA-ILD patients carried the previously reported FPF recurrent mutation [23]: c.1234C>T, p.His412Tyr. The TERT p.His412Tyr MAF is $1.5 \%$ in the European population ExAC database, which could suggest that this variant is a common polymorphism. However, taking into account 1) a MAF of $0.6 \%$ in the overall ExAC database, 2) evidence of linkage of p.His412Tyr to familial pulmonary fibrosis [24] and 3) the functional consequences including shortened telomere length [24], in addition to decreased catalytic activity in vitro $[23,25]$, we considered p.His412Tyr a low penetrant mutation and included it in our genetic association test. RTEL1 sequencing revealed four patients with four heterozygous mutations: three new mutations (c.2695 T>C, p.Phe899Leu; c.2824G>A, p.Asp942Asn; and c.2875C>T, p.His959Tyr) and the previously reported pathogenic mutation: c.2890T $>$ C, p.Phe964Leu [22]. The p.Phe899Leu, p.His959Tyr and p.Phe964Leu mutations were not listed in the ExAC database, but p.Asp942Asn had an ExAC MAF of $2.06 \times 10^{-4}$. One RA-ILD patient carried a PARN heterozygous frameshift mutation

TABLE 1 Demographic and phenotypic characteristics of 101 patients with rheumatoid arthritis (RA)-associated interstitial lung disease (ILD) according to mutation status in familial idiopathic pulmonary fibrosis linked genes

\begin{tabular}{|c|c|c|c|c|}
\hline Characteristic & All patients & With mutations & Without mutations & p-value \\
\hline Age at RA onset years & $53.54 \pm 15.40$ & $45.83 \pm 14.76$ & $54.59 \pm 15.26$ & 0.07 \\
\hline Age at ILD onset years & $61.42 \pm 11.81$ & $53.27 \pm 10.21$ & $62.51 \pm 11.63$ & 0.015 \\
\hline ILD duration years & $5.17 \pm 5.99$ & $5 \pm 3.28$ & $5.19 \pm 6.29$ & 0.87 \\
\hline RA duration preceding ILD detection years & $7.93 \pm 10.83$ & $8 \pm 11.59$ & $7.92 \pm 10.80$ & 0.98 \\
\hline Ever-smoker & $55 / 101(54.5)$ & $7 / 12(58.3)$ & 48/89 (53.9) & 1.00 \\
\hline Active smoker & $9 / 55(16.4)$ & $2 / 7(28.6)$ & $7 / 48(14.6)$ & 0.70 \\
\hline Fibrogenic exposure or airborne contaminants & $26 / 82$ (31.7) & $2 / 10(20)$ & $23 / 72$ (31.9) & 0.69 \\
\hline Methotrexate ever & $82 / 101(81.2)$ & $9 / 12(75)$ & $73 / 89(82)$ & 0.85 \\
\hline Anti-TNF biologic ever & 28/99 (28.3) & $6 / 12(50)$ & $22 / 87(25.3)$ & 0.15 \\
\hline \multicolumn{5}{|l|}{ RA manifestations } \\
\hline ACPA-positive & $78 / 95(82.1)$ & $10 / 11(90.9)$ & $68 / 84(80.9)$ & 0.69 \\
\hline RF-positive & $84 / 99(84.8)$ & $12 / 12(100)$ & $72 / 87(82.8)$ & 0.26 \\
\hline NSIP & $8 / 101(7.9)$ & $1 / 12(8.3)$ & $7 / 89(7.9)$ & \\
\hline Unclassifiable, other & $21 / 101(20.8)$ & $3 / 12(25)$ & $18 / 89(10.2)$ & \\
\hline \multicolumn{5}{|l|}{ Pulmonary function test results at inclusion } \\
\hline FVC \% pred & $85.08 \pm 25.78$ & $76 \pm 27.85$ & $86.28 \pm 25.43$ & 0.27 \\
\hline DLco \% pred & $55.65 \pm 19.47$ & $50.36 \pm 23.65$ & $56.41 \pm 18.85$ & 0.43 \\
\hline TLC \% pred & $80.88 \pm 20.62$ & $77.47 \pm 20.38$ & $81.46 \pm 20.78$ & 0.58 \\
\hline
\end{tabular}

Data are presented as mean \pm SD or (\%), unless otherwise indicated. Some values were missing and the denominators are indicated. TNF: tumour necrosis factor; ACPA: anti-citrullinated peptide antibodies; RF: rheumatoid factor; CT: computed tomography; UIP: usual interstitial pneumonia; NSIP: nonspecific interstitial pneumonia; FVC: forced vital capacity; DLCo: diffusing capacity of the lung for carbon monoxide; TLC: total lung capacity. ${ }^{\#}$ : UIP and possible UIP patterns versus other patterns. 
TABLE 2 Clinical characteristics of 12 rheumatoid arthritis (RA)-associated interstitial lung disease (ILD) patients with familial idiopathic pulmonary fibrosis (FPF)-linked gene mutations

\begin{tabular}{|c|c|c|c|c|c|c|c|c|c|c|c|c|c|c|}
\hline Case & Sex & $\begin{array}{l}\text { Age } \\
\text { years }\end{array}$ & $\begin{array}{c}\text { RA } \\
\text { duration } \\
\text { years }\end{array}$ & $\begin{array}{l}\text { ACPA } \\
\text { status }\end{array}$ & $\begin{array}{c}\mathrm{RF} \\
\text { status }\end{array}$ & $\begin{array}{l}\text { Erosive } \\
\text { disease }\end{array}$ & $\begin{array}{c}\text { Familial } \\
\text { history of } \\
\text { ILD and/or } \\
\text { STS }^{\#}\end{array}$ & $\begin{array}{c}\text { ILD duration } \\
\text { years }\end{array}$ & $\begin{array}{l}\text { Chest CT } \\
\text { diagnosis }\end{array}$ & $\begin{array}{l}\text { FVC } \% \\
\text { pred }\end{array}$ & $\begin{array}{l}\text { DLco \% } \\
\text { pred }\end{array}$ & Locus & c-DNA change & $\begin{array}{l}\text { Amino acid } \\
\text { change }\end{array}$ \\
\hline 1 & $\mathrm{~F}$ & 61 & 3 & Positive & Positive & Yes & No & 3 & UIP & 49 & 33 & PARN & c.1749_1750delAG & p.Ser585fs*5 \\
\hline 2 & $\mathrm{~F}$ & 66 & 14 & ND & Positive & Yes & No & ND & UIP & 44 & NA & RTEL 1 & c. $2695 \mathrm{~T}>\mathrm{C}$ & p.Phe899Leu \\
\hline 3 & M & 66 & 1 & Positive & Positive & Yes & No & 1 & NSIP & 64 & 56 & RTEL1 & c. $2824 \mathrm{G}>\mathrm{A}$ & p.Asp942Asn \\
\hline 4 & M & 64 & 10 & Positive & Positive & Yes & No & 10 & UIP & 58 & 26 & RTEL1 & c. $2875 \mathrm{C}>\mathrm{T}$ & p.His959Tyr \\
\hline 5 & M & 59 & 14 & Positive & Positive & Yes & $\begin{array}{l}\text { Yes (brother } \\
\text { with IPF) }\end{array}$ & 10 & UIP & 50 & 32 & RTEL1 & c. $2890 \mathrm{~T}>\mathrm{C}$ & p.Phe964Leu \\
\hline 6 & $\mathrm{~F}$ & 56 & 5 & Positive & Positive & Yes & $\begin{array}{l}\text { Yes (sister } \\
\text { with IPF) }\end{array}$ & 4 & UIP & ND & 58 & TERT & c. $2383-2 A>G$ & - \\
\hline 7 & $\mathrm{~F}$ & 63 & 38 & Negative & Positive & Yes & No & 5 & Unclassifiable & 102 & 57 & TERT & c. $3323 C>T$ & p.Pro1108Leu \\
\hline 8 & M & 69 & 3 & Positive & Positive & Yes & $\begin{array}{l}\text { Possible } \\
\text { (father with } \\
\text { cirrhosis) }\end{array}$ & 4 & Unclassifiable & 97 & 59 & TERT & c. $1234 C>T$ & p.His412Tyr \\
\hline 9 & M & 68 & 24 & Positive & Positive & Yes & No & 4 & UIP & 104 & 103 & TERT & c. $1234 C>T$ & p.His412Tyr \\
\hline 10 & $\mathrm{~F}$ & 47 & 21 & Positive & Positive & Yes & No & 0 & UIP & 99 & 72 & TERT & c. $1234 C>T$ & p.His412Tyr \\
\hline 11 & M & 48 & 8 & Positive & Positive & Yes & $\begin{array}{c}\text { Yes } \\
\text { (daughter } \\
\text { with IPF) }\end{array}$ & 8 & Unclassifiable & 30 & 60 & TERT & c. $1234 C>T$ & p.His412Tyr \\
\hline 12 & $\mathrm{~F}$ & 40 & 16 & Positive & Positive & Yes & No & 6 & UIP & 28 & 57 & $\begin{array}{l}\text { SFTPC } \\
\text { SFTPC }\end{array}$ & $\begin{array}{l}\text { c. } 218 \mathrm{~T}>\mathrm{C} \\
\text { c. } 180 \mathrm{G}>\mathrm{A}\end{array}$ & $\begin{array}{l}\text { p.lle73Thr } \\
\text { p.Met60lle }\end{array}$ \\
\hline
\end{tabular}

ACPA: anti-citrullinated peptide antibodies; RF: rheumatoid factor; STS: short telomere syndrome; CT: computed tomography; FVC: forced vital capacity; DLCO: diffusing capacity of the lung for carbon monoxide; M: male; F: female; ND: unknown, could not be determined; IPF: idiopathic pulmonary fibrosis; UIP: usual interstitial pneumonia; NSIP: nonspecific interstitial pneumonia. ": familial history of ILD was assessed for all RA-ILD patients carrying mutations in FPF-linked genes; the familial history of STS was assessed for patients carrying mutations in telomere maintenance genes (i.e. TERT, RTEL 1 and PARN). 
(c.1749_1750delAG, p.Ser585fs ${ }^{\star}$ ), not reported in the ExAC database. We found no mutations in TERC, DKC1 or TINF2 genes.

For genes encoding surfactant-related proteins, one RA-ILD patient carried a previously reported heterozygous SFTPC mutation (c.218T>C, p.Ile73Thr) and another carried an unreported SFTPC heterozygous mutation (c.180G >A, p.Met60Ile). Both mutations were located at highly conserved positions in the pro-SP-C-linker domain. One RA-ILD patient was a double heterozygote carrying both a heterozygous mutation in SFTPC (c.218T $>$ C, p.Ile73Thr) and a heterozygous mutation in TERT (c.1234C $>$ T, p.His412Tyr). No mutations were detected in ABCA3 or SFTPA2 genes. Details of the identified mutations are in supplementary table S1.

\section{Predicted structural impact of the TERT and RTEL1 mutations TERT}

His412, located in the telomere RNA-binding domain (TRBD), is predicted to be in a helix involved in the binding of the TERT template-boundary element (TBE), which acts as a molecular guide to position the template in the active site. His412 does not make direct contact with the TBE, but is located on a positively charged TRBD surface (supplementary figure S3), which suggests that the mutation affects binding to structural elements located in the p3 helix and/or pseudoknot. Position 1108 is located on the C-terminal extension (CTE) thumb domain, in the loop that sharply turns the protein chain before the last 24 residues. Amino-acid Pro1108 is located in a local hydrophobic core; leucine substitution at this position preserves the residue's hydrophobic nature and is not predicted to engender major unfolding. However, because proline residues induce a kink in the main chain of the protein, this mutation could destabilise the structure of the terminal residues that interact with the CTE and RNA-template regions, and perhaps the TRBD near His412.

\section{RTEL 1}

RTEL1 encodes an essential iron-sulfur (FeS)-containing DNA helicase that is critical for telomere maintenance and DNA repair. All mutations affect the first RTEL1 harmonin-like domain (supplementary figure S4a and c). Amino acids Phe899 and His959 occupy highly conserved positions. Leucine substitution at position 899 is predicted to disturb interaction with a yet uncharacterised partner of the harmonin-like domain, whereas tyrosine at position 959, although not directly involved in the putative binding groove, might also alter harmonin-like domain interactions. The effect of the asparagine at position 942, located in the $\alpha-3 \alpha-4$ loop, remains undetermined. Furthermore, eucine replacement of phenylalanine at the highly conserved position 964, located in helix $\alpha-5$, is predicted to disturb domain folding [22].

\section{Burden test}

Principal component analysis of WES data genotypes revealed that 81 patients, excluding the 20 outliers among the 101 RA-ILD patients, were clustered with the 1000-genome (1000G) subjects of European ancestry (supplementary figure S5a). Excess mutations in FPF-linked genes were evaluated in these 81 patients and compared to our 1010 controls of European ancestry. The retained patients had an excess number of risk mutations compared to controls: $12.35 \%$ (with at least one candidate disease-associated mutation) versus $4.46 \%$ (burden test, OR $3.17,95 \%$ CI $1.53-6.12 ; \mathrm{p}=9.45 \times 10^{-4}$ ) (figure 1 , table 3 ; supplementary figure S5a). The association remained significant with more stringent clustering on $1000 \mathrm{G}$ individuals of European ancestry: $14.71 \%$ of mutations in 68 cases versus $4.76 \%$ in 903 controls (OR 3.60, 95\% CI 1.72-7.04; $\mathrm{p}=3.14 \times 10^{-4}$ ) (supplementary figure S5b and supplementary table S5).

\section{Genotype-phenotype-association analyses Clinical phenotype}

RA-ILD patients carrying a TERT, RTEL1 or PARN mutation showed no other clinical manifestation related to a telomere syndrome, such as skin abnormalities, typical haematological abnormalities (i.e.macrocytosis, anaemia and thrombocytopenia), bone marrow failure or liver disease. Mean age at ILD onset was significantly lower for patients with mutations than those without mutations: $53.27 \pm 10.21$ versus $62.51 \pm 11.63$ years, respectively; $\mathrm{p}=0.015$ (table 1 ). Plots based on smoothing splines supported a nonlinear association between age at ILD onset and TERT/RTEL1/PARN/SFTPC mutations, with higher mutation probability for patients 36-41 years old than those younger or older $(\mathrm{p}=0.040)$ (figure $2 \mathrm{a}$ ). Results remained significant after removal of the patient with a SFTPC mutation $(\mathrm{p}=0.042)$. No other phenotypic differences were detected; notably, pulmonary function and HRCT chest scan pattern at inclusion were similar for both subgroups (table 1).

\section{Telomere lengths in RA-ILD patients with PARN, TERT or RTEL1 mutations}

Consistent with previously reported telomere lengths of similar mutation carriers [14, 26, 27], telomere lengths in genomic DNA isolated from the circulating leukocytes of 11 RA-ILD patients with TERT, RTEL1 or PARN 
FIGURE 1 Excess mutations in familial pulmonary fibrosis (FPF)linked genes in rheumatoid arthritis-interstitial lung disease (RA-ILD) patients: burden test. Frequencies of TERT, RTEL1, PARN and SFTPC gene mutations in 81 RA-ILD patients and 1010 controls in the French Caucasian population.

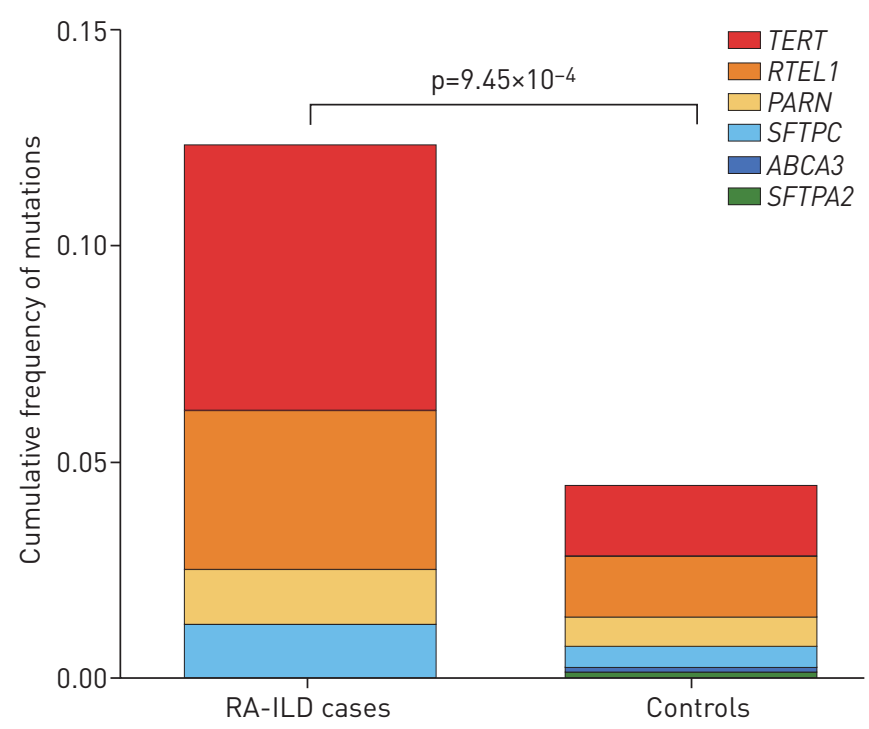

mutations were shorter than those from 15 controls $(\mathrm{p}=0.0114)$ (figure $2 \mathrm{~b}$; supplementary table S2), as confirmed by logistic regression adjusted for age $\left(\mathrm{p}=2.87 \times 10^{-2}\right)$.

\section{Familial history of ILD and short telomere syndrome in patients with mutations}

Among the 12 RA-ILD patients carrying a FPF-linked mutation, three had a family history of interstitial lung disease: case \#5 (RTEL1 p.Phe964Leu) had a brother with idiopathic pulmonary fibrosis (IPF) (deceased); case \#6 (TERT c.2383-2A>G) had a sister with IPF (deceased); and case \#11 (TERT p.His412Tyr and SFTPC p.Ile73Thr) had a daughter with IPF (deceased). The father of case \#8 (TERT p.His412Tyr) died of cirrhosis that was compatible with a short telomere syndrome (table 2) [28].

\section{Discussion}

To date and to our knowledge, this is the first exome sequencing study of RA-ILD patients. Our findings, from a candidate gene approach, provide evidence of an association between RA-ILD and mutations in FPF-linked genes (TERT, RTEL1, PARN or SFTPC). The burden of these mutations was significantly greater in patients than in controls. Moreover, the association was robust after adjustment for more stringent clustering of $1000 \mathrm{G}$ subjects of European ancestry. Our results show that RA-ILD and FPF share genetic risk factors, suggesting common pathogenetic mechanisms. The familial aggregation detected in $25 \%$ of RA-ILD patients carrying at least one mutation in FPF-linked genes supports this hypothesis.

For telomere-maintenance genes, we detected the TERT p.His412Tyr mutation that has been previously linked to FPF and dyskeratosis congenita $[23,25]$. Our findings support the theory of TERT p.His412Tyr as a low penetrance mutation, with two of the four RA-ILD patients carrying the p.His412Tyr mutation, evidently shortened telomere length (supplementary table S2) and one whose father died of cirrhosis compatible with a

TABLE 3 Burden test for 81 rheumatoid arthritis (RA)-associated interstitial lung disease (ILD) patients and 1010 controls among the French Caucasian population

\begin{tabular}{|c|c|c|c|c|c|c|c|c|}
\hline \multirow[t]{2}{*}{ Gene } & \multicolumn{3}{|c|}{ Patients } & \multicolumn{3}{|c|}{ Controls } & \multirow[t]{2}{*}{ p-value } & \multirow{2}{*}{$\begin{array}{c}\text { Odds ratio } \\
(95 \% \mathrm{CI})\end{array}$} \\
\hline & 0 & 1 & 2 & 0 & 1 & 2 & & \\
\hline$A B C A 3$ & 81 & 0 & 0 & 1009 & 1 & 0 & & \\
\hline PARN & 80 & 1 & 0 & 1003 & 7 & 0 & & \\
\hline RTEL1 & 78 & 3 & 0 & 996 & 14 & 0 & & \\
\hline SFTPA2 & 81 & 0 & 0 & 1009 & 1 & 0 & & \\
\hline SFTPC & 80 & 1 & 0 & 1005 & 5 & 0 & & \\
\hline TERT & 75 & 6 & 0 & 993 & 17 & 0 & & \\
\hline Multigene panel testing & 71 & 9 & $1^{\#}$ & 965 & 45 & 0 & $9.45 \times 10^{-4}$ & $3.17(1.53-6.12)$ \\
\hline
\end{tabular}

0: homozygous for the wild-type allele; 1: heterozygous; 2: homozygous for the rare allele. ${ }^{\#}$ : RA-ILD case carrying one SFTPC and one TERT mutation was considered homozygous for a familial pulmonary fibrosis-linked gene mutation. 

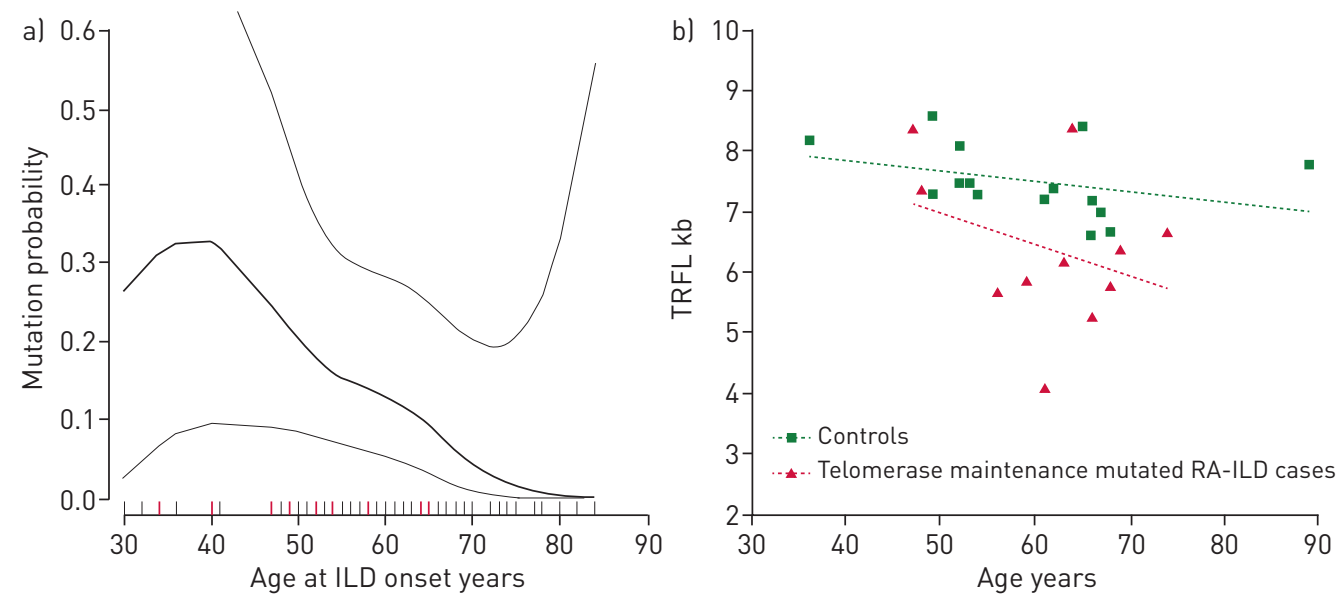

FIGURE 2 Genotype-phenotype association analysis. a) Plots based on smoothing splines of the association between age at interstitial lung disease (ILD) diagnosis and probability of a TERT, RTEL1, PARN or SFTPC mutation. Rheumatoid arthritis-associated ILD (RA-ILD) carriers of a mutation in the multigene panel tested are in red. b) Linear regression analyses of telomere lengths in RA-ILD patients (red) with PARN, TERT or RTEL1 mutation and controls (green). TRFL denotes the telomeric restriction fragment length.

familial short telomere syndrome [28]. Unfortunately, the affected father was not sequenced for TERT, to establish supplementary linkage evidence for TERT p.His412Tyr, which represents one limitation of the present study. We also identified p.Pro1108Leu in a highly conserved residue in the functional domain of the protein and a splice mutation that abolishes the acceptor splice site. Among the three new singletons in RTEL1, p.Phe899Leu, p.His959Tyr and p.Asp942Asn were predicted to be deleterious by at least two of the three prediction tools used. We previously reported the p.Phe964Leu mutation as a possibly deleterious mutation [22]. Furthermore, the PARN mutation leads to a frameshift and premature stop codon. Consistent with previously reported telomere lengths of TERT, RTEL1 or PARN mutation carriers [14, 26, 27], we found shorter lengths in the RA-ILD TERT, RTEL1 or PARN mutation carriers of the present study than in the controls, which confirms the deleterious effects of these mutations on telomere maintenance. The mechanism linking PARN mutations to telomere shortening was recently elucidated: PARN is required for TERC 3'-end maturation [29]. Our RA-ILD patient with the PARN frameshift mutation had the shortest telomeres, which further supports PARN participation in telomere maintenance.

We also investigated genes encoding surfactant-related proteins and detected two SFTPC mutations. The $\mathrm{p}$. Ile73Thr mutation, located within the proSP-C (surfactant protein C) linker domain, accounts for more than $30 \%$ of all SFTPC mutations associated with diffuse parenchymal lung disease in patients with sporadic and inherited (autosomal-dominant) disease [16, 30]. Moreover, p.Met60Ile, a new mutation also located in the non-BRICHOS SP-C domain, was identified. Non-BRICHOS mutations within the proximal $\mathrm{COOH}$ propeptide (e.g. p.Ile73Thr) induce aberrant intracellular trafficking of proSP-C, which eludes cleavage and accumulates in the endosomal system, thereby causing cellular dysfunction [31]. Although we could not examine the functional consequences of the SP-C p.Met60Ile mutation, several lines of evidence favour its pathogenicity: 1) p.Met60Ile is located in a highly conserved region; 2) it has one of the three highest Combined Annotation Dependent Depletion scores; and 3) it is not in the ExAC database.

Our findings demonstrate the usefulness of WES combined with restricted candidate gene analysis in identifying RA-ILD-associated mutations, despite complexities, such as locus heterogeneity and late-onset disease. Because of the small number of available patients, our association study had neither sufficient power nor an appropriate design for gene discovery (e.g. no a priori hypothesis) [32]. Consequently, WES of larger RA-ILD and control populations, probably with international collaboration, is required to identify new RA-ILD risk genes and to refine the exact contribution of FPF-linked genes to the development of RA-ILD.

In the present genetic case-control association study, we provide evidence for an association between a panel of candidate genes (FPF-linked genes) and the "RA-ILD" phenotype, i.e. susceptibility to RA-ILD (RA-ILD versus controls). Our results do not provide information about the putative roles of these genes in 1) susceptibility to overall RA (RA versus controls) and 2) the risk of ILD in the RA population. These issues suggest that a genetic association study should be performed in RA-ILD cases compared to RA cases without ILD. To date, these issues remain unsolved and therefore support the need for an appropriately designed study facilitated by international collaborations, to test whether FPF-linked genes are also RA modifier genes, thereby increasing the risk of ILD in RA. 
From a clinical perspective, the relatively high prevalence of male patients compared to that observed in a recent report of a large multiethnic RA population [33] and the rate of ever smoker patients, are consistent with that previously reported in RA-ILD [19, 34, 35]. Furthermore, consistent with that previously reported for ILD patients with RTEL1 or TERT mutations, ILD occurred earlier in RA-ILD patients with mutations than in those without mutations in telomere-maintenance genes, which might illustrate genetic anticipation, as has been reported in telomere-mediated disorders [36]. Nonetheless, the relatively small sample of RA-ILD patients carrying a mutation limits a genotype-phenotype association analysis, which emphasises the importance of future international collaborative studies on the genetics of RA-ILD.

FPF-risk genes involved in telomere maintenance might be linked to ILD associated with autoimmune diseases, because PARN or RTEL1 mutations have been identified in ILD patients with RA, autoimmune hepatitis, Sjögren's syndrome and more recently systemic sclerosis [22, 26, 37]. This hypothesis is reinforced by diminished telomerase activity and shortened telomere lengths that are apparently connected to premature immunosenescence in various systemic immune-mediated diseases, and more recently by the identification of TERT as a risk gene for systemic lupus erythematosus [38, 39]. In addition, we detected two SFTPC mutations in RA-ILD patients. To our knowledge, SFTPC mutations have only been associated with or linked to interstitial pneumonia, thereby contributing to ILD pathogenesis via endoplasmic reticulum stress in alveolar epithelial cells [16]. For the first time, our results provide evidence of an association between SFTPC mutations and RA-ILD that might contribute to the hypothesis of a pivotal role of the lung in the pathogenesis of RA [40]. Furthermore, our results were observed in European Caucasian patients and would require replication in other populations.

In conclusion, our findings establish, for the first time, shared genetic risk factors between the RA-ILD phenotype and familial pulmonary fibrosis.

\section{References}

1 Turesson C, O'Fallon WM, Crowson CS, et al. Occurrence of extraarticular disease manifestations is associated with excess mortality in a community based cohort of patients with rheumatoid arthritis. J Rheumatol 2002; 29: 62-67.

2 Suzuki A, Ohosone Y, Obana M, et al. Cause of death in 81 autopsied patients with rheumatoid arthritis. J Rheumatol 1994; 21: 33-36.

3 Turesson C, Jacobsson L, Bergstrom U. Extra-articular rheumatoid arthritis: prevalence and mortality. Rheumatology 1999; 38: 668-674.

4 Sihvonen S, Korpela M, Laippala P, et al. Death rates and causes of death in patients with rheumatoid arthritis: a population-based study. Scand J Rheumatol 2004; 33: 221-227.

5 Bongartz T, Nannini C, Medina-Velasquez YF, et al. Incidence and mortality of interstitial lung disease in rheumatoid arthritis: a population-based study. Arthritis Rheum 2010; 62: 1583-1591.

6 Olson AL, Swigris JJ, Sprunger DB, et al. Rheumatoid arthritis-interstitial lung disease-associated mortality. Am J Respir Crit Care Med 2011; 183: 372-378.

7 Doyle TJ, Lee JS, Dellaripa PF, et al. A roadmap to promote clinical and translational research in rheumatoid arthritis-associated interstitial lung disease. Chest 2014; 145: 454-463.

8 Kim EJ, Collard HR, King TE Jr. Rheumatoid arthritis-associated interstitial lung disease: the relevance of histopathologic and radiographic pattern. Chest 2009; 136: 1397-1405.

9 Kim EJ, Elicker BM, Maldonado F, et al. Usual interstitial pneumonia in rheumatoid arthritis-associated interstitial lung disease. Eur Respir J 2010; 35: 1322-1328.

10 Armanios MY, Chen JJ, Cogan JD, et al. Telomerase mutations in families with idiopathic pulmonary fibrosis. N Engl J Med 2007; 356: 1317-1326.

11 Tsakiri KD, Cronkhite JT, Kuan PJ, et al. Adult-onset pulmonary fibrosis caused by mutations in telomerase. Proc Natl Acad Sci USA 2007; 104: 7552-7557.

12 Kropski JA, Mitchell DB, Markin C, et al. A novel dyskerin (DKC1) mutation is associated with familial interstitial pneumonia. Chest 2014; 146: e1-e7.

13 Alder JK, Stanley SE, Wagner CL, et al. Exome sequencing identifies mutant TINF2 in a family with pulmonary fibrosis. Chest 2015; 147: 1361-1368.

14 Cogan JD, Kropski JA, Zhao M, et al. Rare Variants in RTEL1 are associated with familial interstitial pneumonia. Am J Respir Crit Care Med 2015; 191: 646-655.

15 Stuart BD, Choi J, Zaidi S, et al. Exome sequencing links mutations in PARN and RTEL1 with familial pulmonary fibrosis and telomere shortening. Nat Genet 2015; 47: 512-517.

16 van Moorsel CH, van Oosterhout MF, Barlo NP, et al. Surfactant protein C mutations are the basis of a significant portion of adult familial pulmonary fibrosis in a Dutch cohort. Am J Respir Crit Care Med 2010; 182: 1419-1425.

17 Flamein F, Riffault L, Muselet-Charlier C, et al. Molecular and cellular characteristics of ABCA3 mutations associated with diffuse parenchymal lung diseases in children. Hum Mol Genet 2012; 21: 765-775.

18 Wang Y, Kuan PJ, Xing C, et al. Genetic defects in surfactant protein A2 are associated with pulmonary fibrosis and lung cancer. Am J Hum Genet 2009; 84: 52-59.

19 Kelly CA, Saravanan V, Nisar M, et al. Rheumatoid arthritis-related interstitial lung disease: associations, prognostic factors and physiological and radiological characteristics - a large multicentre UK study. Rheumatology 2014; 53: 1676-1682.

20 Assayag D, Lubin M, Lee JS, et al. Predictors of mortality in rheumatoid arthritis-related interstitial lung disease. Respirology 2014; 19: 493-500.

21 Aletaha D, Neogi T, Silman AJ, et al. 2010 rheumatoid arthritis classification criteria: an American College of Rheumatology/European League Against Rheumatism collaborative initiative. Ann Rheum Dis 2010; 69: 1580-1588. 
22 Kannengiesser C, Borie R, Menard C, et al. Heterozygous RTEL1 mutations are associated with familial pulmonary fibrosis. Eur Respir J 2015; 46: 474-485.

23 Alder JK, Chen JJ, Lancaster L, et al. Short telomeres are a risk factor for idiopathic pulmonary fibrosis. Proc Natl Acad Sci USA 2008; 105: 13051-13056.

24 Marchand-Adam S, Diot B, Magro P, et al. Pulmonary alveolar proteinosis revealing a telomerase disease. Am J Respir Crit Care Med 2013; 188: 402-404.

25 Du HY, Pumbo E, Manley P, et al. Complex inheritance pattern of dyskeratosis congenita in two families with 2 different mutations in the telomerase reverse transcriptase gene. Blood 2008; 111: 1128-1130.

26 Stuart BD, Choi J, Zaidi S, et al. Exome sequencing links mutations in PARN and RTEL1 with familial pulmonary fibrosis and telomere shortening. Nat Genet 2015; 47: 512-517.

27 Diaz de Leon A, Cronkhite JT, Katzenstein AL, et al. Telomere lengths, pulmonary fibrosis and telomerase (TERT) mutations. PloS One 2010; 5: e10680.

28 Armanios M, Blackburn EH. The telomere syndromes. Nat Rev Genet 2012; 13: 693-704.

29 Moon DH, Segal M, Boyraz B, et al. Poly(A)-specific ribonuclease (PARN) mediates 3 '-end maturation of the telomerase RNA component. Nat Genet 2015; 47: 1482-1488.

30 Brasch F, Griese M, Tredano M, et al. Interstitial lung disease in a baby with a de novo mutation in the SFTPC gene. Eur Respir J 2004; 24: 30-39.

31 Hawkins A, Guttentag SH, Deterding R, et al. A non-BRICHOS SFTPC mutant (SP-CI73T) linked to interstitial lung disease promotes a late block in macroautophagy disrupting cellular proteostasis and mitophagy. Am Physiol Lung Cell Mol Physiol 2015; 308: L33-L47.

32 Lee S, Emond MJ, Bamshad MJ, et al. Optimal unified approach for rare-variant association testing with application to small-sample case-control whole-exome sequencing studies. Am J Hum Genet 2012; 91: 224-237.

33 Gazal S, Sacre K, Allanore Y, et al. Identification of secreted phosphoprotein 1 gene as a new rheumatoid arthritis susceptibility gene. Ann Rheum Dis 2015; 74: e19.

34 Gochuico BR, Avila NA, Chow CK, et al. Progressive preclinical interstitial lung disease in rheumatoid arthritis. Arch Intern Med 2008; 168: 159-166.

35 Assayag D, Elicker BM, Urbania TH, et al. Rheumatoid arthritis-associated interstitial lung disease: radiologic identification of usual interstitial pneumonia pattern. Radiology 2014; 270: 583-588.

36 Armanios M. Telomerase and idiopathic pulmonary fibrosis. Mutat Res 2012; 730: 52-58.

37 Mak AC, Tang PL, Cleveland C, et al. Brief Report: Whole-Exome Sequencing for Identification of Potential Causal Variants for Diffuse Cutaneous Systemic Sclerosis. Arthritis Rheumatol 2016; 68: 2257-2262.

38 Georgin-Lavialle S, Aouba A, Mouthon L, et al. The telomere/telomerase system in autoimmune and systemic immune-mediated diseases. Autoimmun Rev 2010; 9: 646-651.

39 Sun C, Molineros JE, Looger LL, et al. High-density genotyping of immune-related loci identifies new SLE risk variants in individuals with Asian ancestry. Nat Genet 2016; 48: 323-330.

40 Catrina AI, Ytterberg AJ, Reynisdottir G, et al. Lungs, joints and immunity against citrullinated proteins in rheumatoid arthritis. Nat Rev Rheumatol 2014; 10: 645-653. 